\title{
Volumetric Overload Shocks Cause the Acute Respiratory Distress Syndrome: Building the Bridge Between Physics, Physiology, Biochemistry, and Medicine
}

\author{
Ahmed N Ghanem* \\ Mansoura University, Faculty of Medicine, Egypt \\ *Corresponding author: Ahmed N Ghanem, Mansoura University, Faculty of Medicine, Egypt
}

ARTICLE INFO

Received: 輩 July 17, 2020

Published: 幽 July 28, 2020

Citation: Ahmed N Ghanem*. Volumetric Overload Shocks Cause the Acute Respiratory Distress Syndrome: Building the Bridge Between Physics, Physiology, Biochemistry, and Medicine. Biomed J Sci \& Tech Res 29(1)-2020. BJSTR. MS.ID.004758.

Keywords: Shock; ARDS; MODS; AKI, Oedema; Starling's law; Capillaryinterstitial fluid transfer

\author{
ABSTRACT
}

The acute respiratory distress syndrome (ARDS) was first reported in 1967. Volumetric overload (VO) of 12-14 L reported in every case but not incriminated in patho-aetiology. Although fluid therapy has been suspected and prospective trials reported VO of 3-10 L in surviving ARDS patients, it has not been incriminated. ARDS is attributed to sepsis, has high morbidity, cost, and mortality. How the precise role of $\mathrm{VO}$ in patho-aetiology of ARDS was unraveled and the BRIDGE between physics, physiology, biochemistry, and medicine was built, remain unknown. Here I show how VO complicates fluid therapy inducing shock (VOS) unraveled by critical analytical review of key articles as well as my own research while building the BRIDGE between basic sciences and medicine. The new VOS is of two types: VOS 1 and VOS 2. Hyponatraemia characterizes VOS 1 that is mistaken for a recognized shock and wrongly treated with further volume expansion complicating into VOS 2 causing secondary ARDS. It is known in urology as the transurethral resection of the prostate (TURP) syndrome, induced by $1.5 \%$ Glycine absorption and/or 5\%Glucose infusion for which hypertonic sodium therapy is lifesaving. Sodium-based crystalloids and colloids fluid therapy also induce VOS 2 causing primary ARDS. Both types of ARDS present with multiple organ dysfunction syndromes (MODS). Cerebral features of coma, convulsions and paralysis predominate in VOS 1 or secondary ARDS. Primary VOS 2 causes ARDS in which acute lung injury and acute kidney injury (AKI) predominate. Trunk oedema, coagulopathy and excessive bleeding also occur. Many errors on fluid therapy mislead physicians into giving too much fluid in the resuscitation of shock causing ARDS. The faulty Starling's law dictates the faulty rules on fluid therapy using both liberal and conservative fluid regimen. Here I demonstrate how this culprit has been identified in causing ARDS.

\section{Introduction}

The acute respiratory distress syndrome (ARDS) was first reported in 19671. Volumetric overload (VO) of 12-14 liters (L) reported in every case but not incriminated in patho-aetiology. Although fluid therapy has been suspected [1-8] and prospective trials reported VO of 3-10 L in surviving ARDS patients7,8, it has not been incriminated [2-8]. ARDS is attributed to sepsis [9-12], has a high morbidity, cost and mortality [2-12]. How the precise role of VO in patho-aetiology of ARDS was unraveled and the BRIDGE between physics, physiology, biochemistry, and medicine was built, remain unknown. Here I show how VO complicates fluid therapy during shock resuscitation inducing the new shock (VOS) [13-20] unraveled by critical analytical review of key articles as well as my own research, while building the BRIDGE between basic sciences and medicine. The new VOS is of two types: VOS 1 and VOS 2.

Hyponatraemia characterizes VOS 1 that is mistaken for a recognized shock and wrongly treated with further volume expansion complicating into VOS 2 causing secondary ARDS17-20. It is known in urology as the transurethral resection of the prostate 
(TURP) syndrome [21,22], induced by $1.5 \%$ Glycine absorption and/or 5\%Glucose infusion for which hypertonic sodium therapy is lifesaving [22,23]. Sodium-based crystalloids and colloids fluid therapy also induce VOS 2 causing primary ARDS17-20. Both types of ARDS present with the multiple organ dysfunction syndrome (MODS) [13-17]. Cerebral features of coma, convulsions and paralysis predominate in VOS 1 or secondary ARDS. Primary VOS 2 causes ARDS in which acute lung injury and acute kidney injury (AKI) predominate. Trunk oedema, coagulopathy and excessive bleeding also occur [12-16]. Many errors on fluid therapy [18] mislead physicians into giving too much fluid during the resuscitation of shock causing ARDS [24]. The faulty Starling's law dictates the faulty rules on fluid therapy [25-27] using both liberal and conservative fluid regimen [3-6,28]. Here I demonstrate how this culprit has been identified in causing ARDS.

Identifying and precising the role of volumetric overload (VO) in the patho-aetiology of the acute respiratory distress syndrome (ARDS) is an investigation that spans over the last 39 years. It started with attending the post-mortem (PM) examination of 3 patients who were killed by the transurethral resection of the prostate (TURP) syndrome in 1981. I have done concluded and reported 4 studies, which are: investigating the hydrodynamic of the porous orifice (G) tube [17,25-27], prospective study on 100 TURP patients [21], a case series of 23 patients who had the TURP syndrome [16] and physiologic study on the hind limb of sheep [27]. This was accompanied by critical analytical literature review of all the related issues. These have resulted in many new discoveries in physics, medicine and physiology [29]. The analytical literature review is reported here. The G tube study is highlighted to show how the BRIDGE between physics, physiology, biochemistry, and medicine was constructed. Further updated critical analytical review of the selected landmark articles on ARDS showed that authors have, indeed, suspected "Liberal fluid therapy" regime of resuscitation but never incriminated VO in causing ARDS.

Rangel-Frausto [9] conducted a prospective study on The Natural History of the Systemic Inflammatory Response Syndrome (SIRS) in 1995, wrote: "Equal numbers of patients who appeared to have sepsis, severe sepsis, and septic shock but who had negative cultures. They had been prescribed empirical antibiotics for a median of 3 days. The cause of SIRS in these culture-negative populations is unknown, but they had similar morbidity and mortality rates as the respective culture-positive populations". This is true. So, sepsis is either not there at all in half the ARDS patients or put under control by the appropriate and adequate antibiotics in the other half. This indeed, suggest that sepsis and septic shock in the path-aetiology of ARDS are as innocent as the wolf in Josef' story! We know for sure that the wolf did not eat Josef. It is true that sepsis may attack ARDS patient later doing its nasty work inducing the markers of SIRS. We need to look and search harder in order to recognize the hidden culprit not only responsible for causing ARDS in the negative culture group but also among the positive culture group. Is there alibi for the absence of sepsis in causing ARDS? Indeed, there is.

Who is better to testify than Angus and van der Poll10 (2013)? They wrote in the introduction paragraph to the comprehensive article on severe sepsis and septic shock in support of the absence alibi for sepsis as in Josef and wolf theory:

"However, with the advent of modern antibiotics, germ theory did not fully explain the pathogenesis of sepsis: many patients with sepsis died despite successful eradication of the inciting pathogen. Thus, researchers suggested that it was the host, not the germ that drove the pathogenesis of sepsis." With that in mind, particularly as we trust modern antibiotics eradicating sepsis, we continue our search for the real culprit underlying the cause of ARDS. Wioedemann et al. [4] in the FACCT Trial stated in conclusion: "Although there was no significant difference in the primary outcome of 60-day mortality, the conservative strategy of fluid management improved lung function and shortened the duration of mechanical ventilation and intensive care without increasing non-pulmonary organ failures. These results support the use of conservative strategy of fluid management in patients with acute lung injury." This is indeed a sound advice in support of the conservative strategy of FT, but we need to identify and recognize the culprit causing ARDS that is deeply rooted. Showing no significant difference in the primary outcome of 60 -day mortality between the 2 fluids regimes, where there should be one, suggest that the correct Time for detecting the significant difference in mortality is important. Certainly, it is not at 60 or 90 days. It is much earlier than that as shall be explained later.

The role of Starling's forces played in this situation is merely hinted at by Sibbald et al. [30] and Rodney 2010 [31]. These authors are getting closer to solving the puzzle of ARDS, but they only need to know about my work demonstrating Starling's law has proved wrong [17]. Other authors have reported discontent with Starling's law criticizing it from different angles such as Alphonsus and Rodseth's in 2014 [32] and Woodcock and Woodcock [28] excellent reviews on endothelial glycocalyx. They highlight important issues which are of relevance here: the relation to Starling's forces, the importance of a smooth endothelial surface for the capillary and vascular bed normal function and the role of hypervolaemic state in the pathogenesis of ARDS. Robert Hahn reported an article on adverse effects of crystalloid and colloid fluids in 2017 [33], and on understanding volume kinetics in 2020 [34]. I observed 38-years ago the adverse effect of irregular internal surface of the of the $\mathrm{G}$ tube on the negative side pressure and chamber pressure of the G-C circulatory phenomenon particularly when connected to a circulatory system, as well as the effects of volumetrically overloading the system (. I shall return to discuss Starling's forces later but, by now what appeared to be heterogeneous and disjointed evidence at first look, the BRIDGE is shaping up and making sense. 
Montegomery et al. [2] concluded: "Most deaths in the first 3 days after entry into the study could be attributed to the underlying illness or injury. The majority of late deaths were related to sepsis syndrome. Of the 22 patients with ARDS who died after 3 days, 16 (73\%) met our criteria for sepsis syndrome. There was a six-fold increase in sepsis syndrome after ARDS compared with that in the control group $(\mathrm{p}<0.001)$. When sepsis syndrome preceded the ARDS, the abdomen was the predominant source, but when sepsis syndrome occurred after the onset of ARDS there was usually a pulmonary source" Based on this sound research we should segregate those patients who die within 3 days starting at hospital admission from those who die afterwards in order to show the significance in mortality on comparing further subgroups of the studied patients, as shown later. This is important for revealing the culprit causing ARDS. All the action and most of the mortalities of ARDS occur at the time of resuscitation with fluids (the 6-hours period of EGDT) and perhaps up to 3 days, maximum one week, when significant difference in morbidity and mortality may be detected. This is the time period to look for the cause of ARDS and its significant effect on mortality endpoint not the 60 or 90-days' time endpoint. I shall further discuss the significance and importance of Time a little later. Sepsis becomes responsible for the mortality of ARDS after 3 days from onset or hospital admission.

Schuller et al asked an important and excellent question in the title of their report in 1991 [3]: "Is fluid balance during pulmonary oedema a marker or a cause of poor outcome? The answer is in their conclusion: "These data support the concept that positive fluid balance per se is at least partially responsible for poor outcome in patients with pulmonary oedema and defend the strategy of attempting to achieve a negative fluid balance if tolerated hemodynamically." That is correct and most useful advice, but what is causing ARDS? Dr Schuller is saying that positive fluid balance per se that is VO or hypervolaemia is at least partially responsible. I agree and go further perhaps it may be even totally responsible. Please, follow his advice of "attempting to achieve a negative fluid balance" and trust the haemodynamic of the cardiovascular system (CVS) will sort itself out spontaneously and return to normal.

The prevalence of "liberal" fluid infusion in resuscitation of all types of shocks not only septic shock in clinical practice all over the world is attributed to an impactful article by Rivers et al, reported at The N Engl J Med 2001 [11]. Dr Rivers' investigation reported the Early Goal-Directed Therapy (EGDT) in the treatment of severe sepsis and septic shock. In this single-centre study published more than 19 years ago involving patients presenting to the emergency department with severe sepsis and septic shock, the conclusion was: "mortality was markedly lower among those who were treated according to a 6-hour protocol of EGDT, in which intravenous fluids, vasopressors, inotropes, and blood transfusions were adjusted to reach central hemodynamic targets, than among those receiving usual care" Usual care means conservative fluid regime. There is something grossly wrong with this conclusion, but I cannot tell what is it? Not yet. Let us see what other author investigators have said first. The EGDT of liberal fluid infusion has been termed "aggressive" by some authors. However, it has been adopted all over the world not only for the therapy of septic shock but also whenever fluid therapy is required.

In another article by Dr Rivers 10 years later in 2012 [12] he compared the liberal to the conservative approach concluding in his last statement: "In contrast to what is true in politics, in fluid management of acute lung injury, it is $\mathrm{OK}$ to be both liberal and conservative." So, Dr Rivers says it is OK to have it both ways: "one for the ebb and one for the flow"! Sorry, sir, I disagree. It is not OK. It is not politics either. No, you cannot have it both ways. The right way is only one. The issue here is how much fluid should be infused during the ebb phase of shock and does it have a maximum limit? Replace the loss but do not overdo it. Since the CVS' maximum capacity of an adult is $7 \mathrm{~L}$ and the normal blood volume is $5 \mathrm{~L}$, the maximum infused volume of fluid should be limited by the maximum capacitance of the CVS. What do you expect when you try to fit 10-15 L of fluid into a $7 \mathrm{~L}$ capacity container? Simple physics and common sense indicate that it must spell over if it is open system or burst if closed! The cardiovascular system is no exception. Dr Rivers should re-examine his own data and tell us where and why he went so grossly wrong.

The traumatized bleeding patient, for example, dies before reaching the hospital if half the blood volume quickly lost. In sepsis, severe sepsis and septic shock, there is no fluid loss at all. In the "liberal" approach of EGDT there seem to be no limit on how much fluid should be infused. This VO induce hypervolaemic state that internally drowns the swollen patient on ICU. To resolve this most elusive puzzle of ARDS, there is a need to define this maximum volume of infused fluids not only for the liberal but also for the conservative approach. I know there are a few situations when this maximum must be exceeded such as in burned and the continuously bleeding patients that cannot be stopped and perhaps heat stroke. In these situations, the advice is: stop the bleeding, replace the loss but do not overdo it.

The PRISM Investigators reported its Trial by Rowan et al at NEJM 20177 concluded: "In this meta-analysis of individual patient data, EGDT did not result in better outcomes than usual care and was associated with higher hospitalization costs across a broad range of patient and hospital characteristics." Thank you, Dr Rowan and colleagues for the excellent research and report. This is good evidence-based medicine, but more is needed, from you, and you have the data to provide it. Based on this conclusion that agrees with other multi-centre trials I wonder is time to say goodbye Dr, Rivers? The aggressive and deleterious liberal approach of EGDT is no longer wanted. It should be abandoned immediately. Even when the nasty liberal approach goes away, hopefully soon, it 
remains bad enough with the conservative regime as it is now that must be sorted out! I wonder what Dr Rivers has to say about this, particularly as authors of 3 other huge prospective multi-centre trials of The ProCESS/ARISE/ProMISe reported similar conclusion by Huang et al. [8].

What is the gain from doing such long expensive trials if its conclusion and recommendation are not implemented, immediately? What is most interesting, relevant, and important in the reported data in the results section of the article by Rowan et al. 2017 [7] on fluid balance in ARDS patients who wrote the following: "Each study day the liberal-strategy group received more fluid than the conservative-strategy group and on days 1 through 4 had a lower urinary output, resulting in a higher cumulative fluid balance (Table 1). During the study, the seven-day cumulative fluid balance was $-136 \pm 491 \mathrm{ml}$ in the conservative-strategy group, as compared with $6992 \pm 502 \mathrm{ml}$ in the liberal-strategy group $(\mathrm{P}<0.001)$ (Figures $1 \&$ 2 of the Supplementary Material). For patients who were in shock at baseline, the cumulative seven-day fluid balance was $2904 \pm 1008$ $\mathrm{ml}$ in the conservative-strategy group and $10,138 \pm 922 \mathrm{ml}$ in the liberal-strategy group $(\mathrm{P}<0.001)$. For patients who were not in shock at baseline, the cumulative fluid balance was $-1576 \pm 519 \mathrm{ml}$ in the conservative-strategy group and $5287 \pm 576 \mathrm{ml}$ in the liberalstrategy group $(\mathrm{P}<0.001)$."

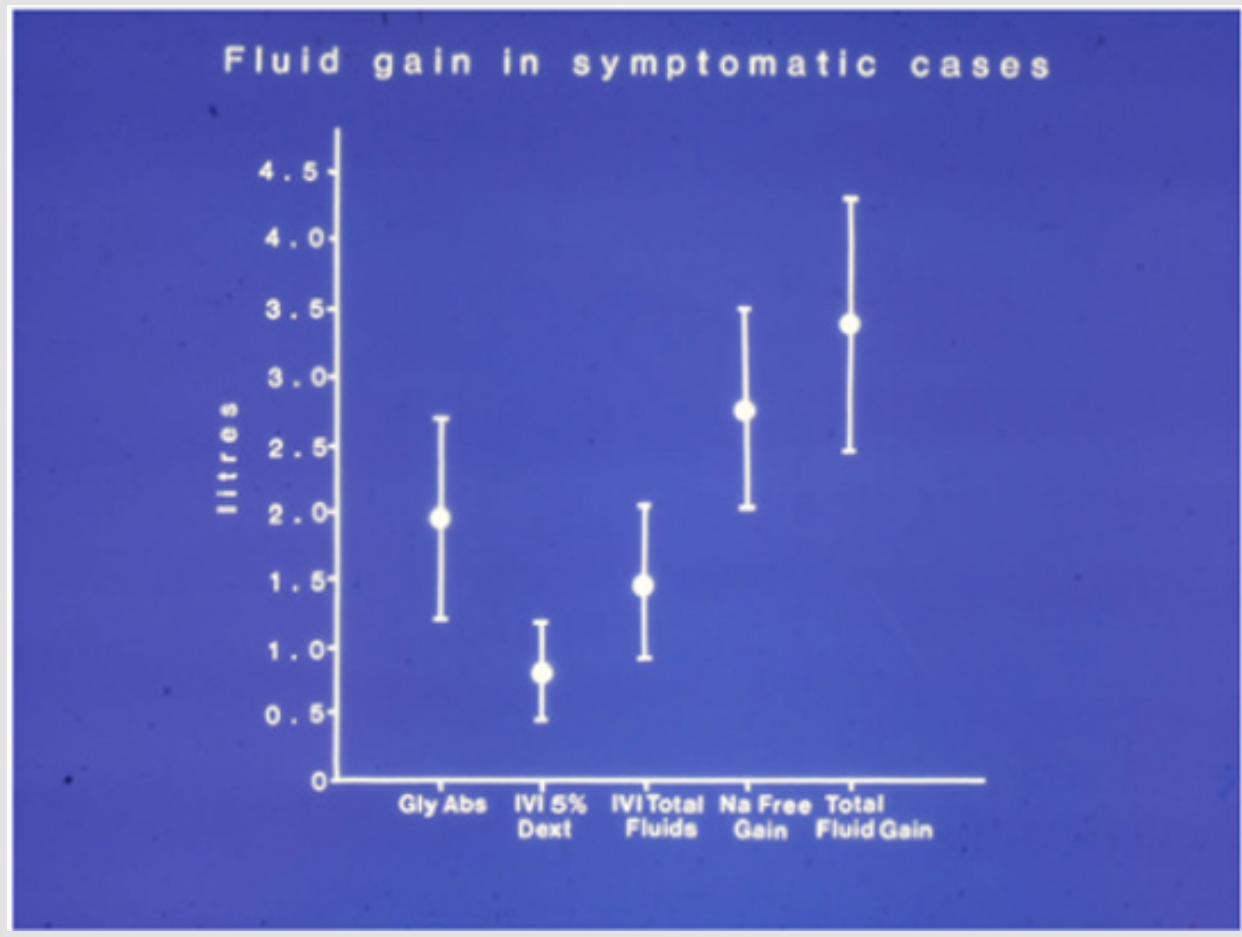

Figure 1: The means and standard deviations of volumetric overload in 10 symptomatic patients presenting with shock and hyponatraemia among 100 consecutive patients during a cohort prospective study on transurethral resection of the prostate. The gained fluids were Glycine absorbed (Gly abs), intravenously infused 5\% Dextrose (IVI Dext) Total IVI fluids, Total Sodiumfree fluid gained (Na Free Gain) and total fluid gain in Liters. A mean of 3.5 ( $p=0.0001)$ liters VO occurred in symptomatic patients presenting with hypotensive shock, recognized as VOS1. 


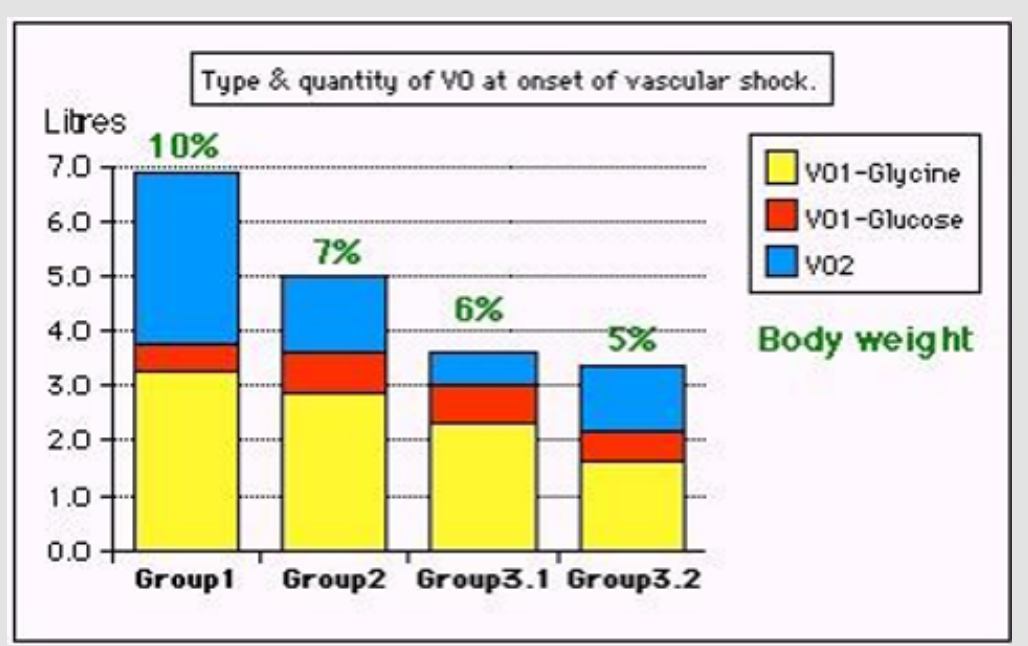

Figure 2: Volumetric overload (VO) quantity (in liters and as percent of body weight) and types of fluids. Group 1 was the 3 patients who died in the case series as they were misdiagnosed as one of the previously known shocks and treated with further volume expansion based on the conservative approach. They had the clinical picture of VOS 2 and ARDS. Group 2 were 10 patients from the series who were correctly diagnosed as volumetric overload shock (VOS 1) and treated with hypertonic sodium therapy (HST). Group 3 were 10 patients who were seen in the prospective study and subdivided into 2 subgroups; Group 3.1 of 5 patients treated with HST and Group 3.2 of 5 patients who were treated with "guarded" volume expansion using isotonic crystalloids/plasma/blood of the standard conservative approach but much less volume- being "Guarded". One patient from the conservatively treated group 3.2 developed ARDS with coma, convulsions, and bizarre paralysis on the second postoperative day. He was seen by a Neurologist and diagnosed as cerebrovascular accident. He fully and immediately recovered from ARDS, coma and paralysis after belated treatment with $\mathrm{HST}$ using $5 \% \mathrm{NaCl}$ and $8.4 \% \mathrm{NaCo3}$. After HST the patient recovered fully from AKI, ARDS, Coma and paralysis. Recovery from AKI that was unresponsive to loop diuretics occurred with the infusion of HST after passing 4.5L of urine. Groups 2 and 3.1 responded similarly to HST. (Reproduced with the permission of author from open access journal).

Table 1: The manifestations of VOS 1 of the TURP syndrome for comparison with ARDS manifestations induced by VOS2.

\begin{tabular}{|c|c|c|c|c|}
\hline Cerebral & Cardiovascular & Respiratory & Renal & Hepatic \& GIT \\
\hline Numbness & Hypotension & Cyanosis & Oliguria & Dysfunction: \\
Tingling & Bradycardia & FAM $^{4}$ & Annuria $^{8}$ & Bilirubin $\uparrow$ \\
SBB & SGOT $\uparrow$ \\
COC2 & Dysrhythmia & APO $)^{5}$ & Alkaline Phosph \\
Convulsions & Renal failure or & GIT symptoms \\
Coma & CV Shock* & Arrest & AKI $^{9}$ & DGR \\
PMBCI 3 & Cardiac Arrest & CPA & Urea $\uparrow$ & Paralytic ileus \\
& Sudden Death & Shock lung & Creatinine $\uparrow$ & Nausea \& Vomiting. \\
\hline
\end{tabular}

$\begin{array}{ll}\mathrm{SBB}^{1} & \text { Sudden bilateral blindness } \\ \mathrm{COC}^{2} & \text { Clouding of consciousness }\end{array}$

$\mathrm{PMBCI}^{3}$ Paralysis mimicking bizarre cerebral infarctions, but is recoverable on instant use of $\mathrm{HST}$ of $5 \% \mathrm{NaCl}$ and/or $\mathrm{NaCo}$, and so is coma and AKI

$\begin{array}{ll}\mathrm{FAM}^{4} & \text { Frothing around the mouth } \\ \mathrm{APO}^{5} & \text { Acute pulmonary oedema } \\ \mathrm{RA}^{6} & \text { Respiratory arrest } \\ \mathrm{CPA}^{7} & \text { Cardiopulmonary arrest }\end{array}$

ARDS $^{\$}$ Acute respiratory distress syndrome, occurs later on ICU

$\mathrm{AKI}^{9} \quad$ Acute kidney injury

DGR $^{10} \quad$ Delayed gut recovery

CV Shock* Cardiovascular shock of VOS reported here as VOS 1 and VOS2.

Annuria $^{8} \quad$ That is unresponsive to diuretics but responds to HST of $5 \% \mathrm{Ncl}$ and $/$ or $8.4 \% \mathrm{NaCo} 3$

$\mathrm{AKI}^{8} \quad$ Acute kidney injury

Also occurs the excessive bleeding at the surgical site and

Leucocytosis occurred in the absence of sepsis and septic shock 
Thank you very much indeed Kathryn for documenting these data. Thanks also to Haung et al. [8] and all authors of the ProCESS/ARISE/ProMISe trials for having similar results. In my knowledge, this is the first time we come across a prospective trial on ARDS that reports VO data with such clarity except the original first report on ARDS by Ashboagh et al. [1]. However, no article has incriminated VO in the patho-aetiology of ARDS except our reports mentioned in all the self-references reported here. Now we are talking business. I have underlined the key words for the reader to have a deeper thought about it while commenting on it. So: The cumulative volumetric overload (VO) was $-136 \pm 491 \mathrm{ml}$ in the conservative-strategy group, as compared with $6992 \pm 502 \mathrm{ml}$ in the liberal-strategy group $(\mathrm{P}<0.001)$. For patients who were in shock at baseline, the cumulative seven-day VO was $2904 \pm 1008 \mathrm{ml}$ in thconservative-strategy group and $10,138 \pm 922 \mathrm{ml}$ in the liberalstrategy group $(\mathrm{P}<0.001)$.

For patients who were not in shock at baseline, the cumulative VO was $-1576 \pm 519 \mathrm{ml}$ in the conservative-strategy group and $5287 \pm 576 \mathrm{ml}$ in the liberal-strategy group $(\mathrm{P}<0.001)$. First, the negative sign (-) indicating negative fluid balance has appeared in the data above and is very important. It characterizes the nonsymptomatic patients among the conservative-strategy group. These patients should be used as the controls for the statistical analysis of the data. I have been waiting for 39 years to see these VO results. I am still waiting to see VO data with statistical significance in mortality patients. I plead with and urge the respected authors of major randomized Trials of FACCT, PRISM, ProCESS, ARISE, and ProMISe to come forward with these data, please, as based on the statistical analysis suggested below.

Before proceeding, may I ask Dr Rowan, where is VO data for the dead patients, please? Angus and van der Poll (2013) [10] did an individual patient meta-analysis review article at NEJM also concluded no significant effect of early goal directed therapy (EGDT) on mortality with increased cost and ICU bed stay. Furthermore, if statistics programs can talk, they may say: These VO quantities of $>3$ $\mathrm{L},>5 \mathrm{~L}(7 \%)(\mathrm{P}<0.001)$ and $>10 \mathrm{~L}$ (about 15\%) body weight (BW) $(\mathrm{P}<0.001)$ underlined above, retained in all surviving ARDS patient is relevant and highly significant in relation to patho aetiology of ARDS as well as its morbidity. These figures are staggering cumulative $\mathrm{VO}$ of retained fluid that indeed drowns the swollen patient internally and causes ARDS morbidity and mortality.

Again, the VO data or the amount of retained fluid at the time of death was lacking. If you do have it, please publish it or send it to me. There is nothing to be afraid or ashamed of as it happens with every physician involved in fluid therapy in hospitals all over the World. So, it sounds like normal, but it is not. I am well prepared to defend you with body and soul against anyone who dare to accuse you of fluid overloading your patient. This is the usual standard practice of every physician, surgeon, anaesthetists and intensivist in every hospital all over the globe who use not only the liberal regime of EGDT but also the conservative regime of Bolus Fluid Therapy
(BFT). No exceptions made. For anyone using the offensive term accusation of fluid overloading a patient, I say this: what scientific basis your practice on fluid therapy is based on? And, do you have evidence from your own practice to show that you can do better than your colleagues? If you do let us examine it!

A retained volume of $10 \mathrm{~L}$ must be found in a very sick ARDS patient indeed. Perhaps VO of $>12-14 \mathrm{~L}$ reported by Ashbaugh et al1 is the VO that characterizes the dead patients. Even the $3 \mathrm{~L}$ volume of fluid that is the normal daily fluid intake becomes pathological when infused to a normal adult within one hour. Professor Robert Hahn from Sweden has done lots of research infusing various types of fluid used in clinical practice to normal adult volunteers, and reported $>340$ articles on the TURP syndrome alone (PubMed 2017) and 506 articles in total (PubMed search on 10/02/2020): Here is what Robert Hahn, Professor of Anaesthesia and Head of the National Fluid Academy (NFA), concluded in abstract of a recent review article in 2017 [33]: "Guidelines for fluid therapy rarely take into account that adverse effects occur in a dose-dependent fashion. Adverse effects of crystalloid fluids are related to their preferential distribution to the interstitial of the subcutis, the gut, and the lungs. The gastrointestinal recovery time is prolonged by 2 days when more than 2 liters is administered. Infusion of 6-7 liters during open abdominal surgery results in poor wound healing, pulmonary oedema, and pneumonia. There is also a risk of fatal postoperative pulmonary oedema that might develop several days after the surgery. Even larger amounts cause organ dysfunction by breaking up the interstitial matrix and allowing the formation of lacunae of fluid in the skin and central organs, such as the heart. Adverse effects of colloid fluids include anaphylactic reactions, which occur in 1 out of 500 infusions. The possibility that hydroxyethyl starch causes kidney injury in patients other than those with sepsis is still unclear. For both crystalloid and colloid fluids, coagulation becomes impaired when the induced haemodilution has reached $40 \%$. Coagulopathy is aggravated by co-existing hypothermia. Although oedema can occur from both crystalloid and colloid fluids, these differ in pathophysiology."

Thank you, Professor Hahn for a most impressive work indeed. New guidelines based on currently available evidence on fluid therapy for resuscitation of sepsis, septic shock, trauma patients, critically ill patients, ARDS and patients undergoing prolonged major surgery are badly needed. The underlining of text is mine to discuss and bald text is for the benefit of reader to think about. Professor Hahn is the expert witness on fluid therapy.

Now let us look at the importance of (Time) of VO occurrence. The report from the PRISM Investigators by Dr, Rowan [7] stated: "our patients received their first protocol intervention an average of 43 hours after admission to the ICU and 24 hours after meeting the criteria for acute lung injury." The onset of ARDS occurs at the Time of resuscitation, which starts on arrival to accident and emergency department for trauma patients, during anaesthetic induction, in the operating theatre during prolonged major surgery 
and on ICU affecting both the liberal and conservative groups. In the conservative group it is induced by the BFT and in the liberal group it is induced by EGDT endpoint. This occurs during the first 6-hours period of EGDT or BFT when most of the VO has already occurred in both liberal and conservative groups. That is the reason for finding that there is no significant difference in mortality between the 2 groups at 60- and 90-day endpoint, because it is much too late. However, it is possible to detect a highly significant VO in relation to the morbidity and mortality of ARDS patients on re-analyzing the currently available data from trials such as PRISM (2017) [7], ProCESS, ARISE, and ProMISe (2013) [8] and FACCT (2006) [4].

This can be achieved if each of the two groups of liberal and conservative are subdivided into 3 subgroups of asymptomatic (A), symptomatic with morbidities (B) and the dead or mortalities (C) subgroup. The mean VO for each of the subgroup A, B and C is calculated and statistically analyzed: comparing those with morbidity $\mathrm{B}$ and mortality $\mathrm{C}$ with the non-symptomatic $\mathrm{A}$ group acting as controls. The calculation of the fluid balance VO should be done at the end of resuscitation, at the end of the 6-hour period of EGDT or after surgery, at 3-days later, and at 7- day endpoint times, and on the day of death. Ideally it should be done on daily basis from the day of admission to the hospital accident and emergency department till death or discharge. The calculated VO of retained fluid should be augmented by the daily changes in the patient's body weight occurring during the hospital stay. So, the volumetric and gravimetric data is made available. The investigation by Rowan7 started a day or two after the onset of ARDS thus the data of the 6-hour period may not be available. The data at the start of the trial will do for now. I expect highly significant result associating VO with both the morbidity and mortality of ARDS with a $(\mathrm{P}=0.0001)$ as our research demonstrated in the other type of VO of sodium-free fluid type1 (VO1) such as 5\% Glucose and/or $1.5 \%$ Glycine inducing VO shock type 1 (VOS 1) This is known in Urology as the TURP syndrome21,22 or hyponatraemic shock first reported by Harrison III et al 195622. Multiple regression analysis in our prospective study proved $\mathrm{VO}$ as the most significant factor in relation to clinical picture and patho aetiology of TURP syndrome and secondary ARDS ( $\mathrm{p}=0.0001)$.

THE VO and fluid type of the 10 symptomatic patients among the 100 patients of the prospective trial is reported [21]. There were no mortalities in our prospective study but there was morbidity affecting 10 (10\%) patients, one of whom among the conservatively treated group developed the criteria of secondary ARDS who suffered coma, next day, with convulsions and paralysis diagnosed by a neurologist as cerebrovascular accident. He was crossed over and belatedly treated with HST with complete cure. Morbidities of Secondary ARDS is shown in Table 1. Data for the 23 cases series is shown in (Table 2). In our study, a mean total VO of $\pm 3.5 \mathrm{~L}$ was gained by 10 symptomatic patients This gained $\pm 3.5 \mathrm{~L}$ included $0.5 \mathrm{~L} 5 \%$ Glucose and $1 \mathrm{~L}$ saline or Hartmann's, and $2 \mathrm{~L}$ of $1.5 \%$ Glycine absorbed during the TURP surgery. The total fluid gain in symptomatic patients of $3.5 \mathrm{~L}$ compared with $1.36 \mathrm{~L}$ in asymptomatic patients was significant $(p=0.0001)$. The VO of 7 L $(10 \%$ BW $)$ of the case series characterizes dead ARDS patients who presented initially with VOS 1 of TURP syndrome and later with secondary ARDS (Table 2). The VO of $5 \mathrm{~L}(7 \% \mathrm{BW})$ was that of patients presented with severe morbidity of VOS 1 or secondary ARDS.

Table 2: The data of the 23-patients of the case series study [11]; the second clinical study on which this article is based. The significant changes of serum solute contents are shown in bald font with the corresponding p-value. Most of the patients showed manifestation of ARDS (Table 1) of which the cerebral manifestation predominated, being on initial presentation (Regional Anaesthesia) and representation of VOS 1 (General Anaesthesia). However, most patients were given large volume of saline that elevated serum sodium to near normal while clinical picture became worse. They suffered VOS2 that caused ARDS. The VO of patients to whom these data belongs are shown. Please note the elevation of urea and unurea of Group 1 who died indicated AKI. Elevations oh Bilirubin and AST indicated hepatic dysfunctions. White cell count (WCC) elevation indicated inflammatory response of VOS 2 in ARDS or SIRS in the absence of sepsis.

\begin{tabular}{|c|c|c|c|c|c|c|c|c|}
\hline & A & B & C & D & $\mathbf{E}$ & $\mathbf{F}$ & G & $\mathbf{H}$ \\
\hline 1 & & Gr 1 & Gr 2 & Gr 3 & Gr 3.1 & Gr 3.2 & Normal & Units \\
\hline 2 & $\begin{array}{l}\text { Number of } \\
\text { patients }\end{array}$ & 3 & 10 & 10 & 5 & 5 & Mean & \\
\hline 3 & Age & 71 & 70 & 75 & 72 & 78 & 72 & Year \\
\hline 4 & Body Weight (BW) & 69 & 70 & 68 & 71 & 65 & 69 & $\mathrm{~kg}$ \\
\hline 5 & \multicolumn{6}{|c|}{ Postoperative serum solute concentrations: } & \multicolumn{2}{|c|}{ Preoperative } \\
\hline 6 & Osmolality & 271 & 234 & 276 & 282 & 271 & 292 & mosm/1 \\
\hline 7 & $\mathrm{Na}+$ & 110 & 108 & 120 & 119 & 121 & 139 & $\mathrm{mmol} / 1$ \\
\hline 8 & $\mathrm{Ca}++$ & 1.69 & 1.79 & 1.85 & 1.84 & 1.86 & 2.22 & $\mathrm{mmol} / 1$ \\
\hline 9 & $\mathrm{~K}+(\mathrm{P}<.05)$ & 5.6 & 4.8 & 5.0 & 4.9 & 5.0 & 4.46 & $\mathrm{mmol} / 1$ \\
\hline 10 & $\mathrm{Co} 2(\mathrm{P}=.002)$ & 23.0 & 23.0 & 25.5 & 24.0 & 26.4 & 27.30 & $\mathrm{mmol} / 1$ \\
\hline 11 & Glucose & 13.2 & 17.3 & 16.4 & 15.9 & 16.9 & 6.20 & $\mathrm{mmol} / 1$ \\
\hline 12 & Urea $(\mathrm{P}=.0726)$ & 26.5 & 9.0 & 6.6 & 6.8 & 6.4 & 6.7 & $\mathrm{mmol} / 1$ \\
\hline 13 & Bilirubin $(\mathrm{P}<.05)$ & 19 & 16 & 8 & 6 & 9 & 7 & $\mathrm{mmol} / 1$ \\
\hline
\end{tabular}




\begin{tabular}{|c|c|c|c|c|c|c|c|c|}
\hline 14 & AST & 124 & 32 & 20 & 18 & 21 & 20 & $\mathrm{mmol} / 1$ \\
\hline 15 & Protein & 43 & 52 & 48 & 44 & 52 & 62 & $\mathrm{~g} / 1$ \\
\hline 16 & Albumin & 23 & 30 & 30 & 28 & 32 & 39 & $\mathrm{~g} / 1$ \\
\hline 17 & Hb $(\mathrm{P}=.0018)$ & 119.3 & 127.9 & 114.5 & 105.2 & 123.8 & 138.8 & $\mathrm{~g} / 1$ \\
\hline 18 & WCC $(\mathrm{P}<.005)$ & 18.9 & 16.2 & 7.5 & 7.8 & 7.2 & 8.0 & $\mathrm{per} \mathrm{HPF}$ \\
\hline 19 & Glycine & & & 10499 & & & 293 & $\mu \mathrm{mol} / 1$ \\
\hline 20 & Therapy & CT & HST & Random: & HST & CT@ & & \\
\hline 21 & Outcome & Death & Full Rec. & & Full Rec. & Morb. @ & \\
\hline
\end{tabular}

Luckily, the TURP syndrome is characterized with acute dilution HN. This allowed recognizing VOS 1 which in turn allowed recognizing VOS 2 of both secondary and primary types. Such VOS 1 patients present in theatre with shock that is usually mistaken for septic or haemorrhagic shock and wrongly treated with further volume expansion using isotonic sodium-based fluids (of crystalloids or colloids and/or blood) which erase HN and transfer TURP syndrome into secondary ARDS. The patient may even present with cardiac or respiratory or cardiopulmonary arrest, when more sodium-based fluids are infused, thus VOS 1 is transferred to secondary VOS 2 causing ARDS. The first 3 patient of group 1 who fulfilled the typical criteria of ARDS of secondary VOS 2 presented with shock in theatre, were mistaken for a haemorrhagic or septic shock and were wrongly treated with further volume expansion then developed ARDS and died. Their post-mortem was attended. The vital organs of lung, heart, brain and liver were oedematous, and the patient's trunk was grossly swollen and there was accumulation of $3 \mathrm{~L}$ of fluids in the peritoneal cavity. The gut was loaded with fluid and there was a volume in both pleural spaces of $1.5 \mathrm{~L}$ each. When the pathologist, doing the PM examination, was asked in 1981: "Why don't you include this internal drowning in your report?" His reply was: "Because it offends the treating physicians!"

So, does the label of "fluid overload" that puts the blame on the treating physician but in fact it is the endpoint of EGDT in the liberal approach, and the BFT based on the faulty rules on FT in the conservative approach are to blame! Underlying both is the culprit faulty Starling's law which dictates the faulty rules on fluid therapy. Hence, I recommend that the offensive term of "fluid overload" to be replaced with either VO or hypervolaemia, or volume kinetics proposed by Hahn instead. There is no doubt that VOS and ARDS are iatrogenic complication of fluid therapy but unrecognized and underestimated. Further experience with 16 patients of the case series study who presented with TURP syndrome first and secondary ARDS later were successfully treated and saved from certain death by hypertonic sodium therapy (HST) of $5 \% \mathrm{NaCl}$ and/ or $8.4 \% \mathrm{NaCo} 3$ infusion. The other 4 patients of the conservatively treated group were guarded against further VO of conservative therapy but had morbidities (Table 1). Recovery from AKI and coma of TURP or ARDS using HST was instant with full immediate recovery at the end of the one hour HST infusion by producing 4-5 $\mathrm{L}$ of urine through the urinary catheter and waking up from coma- asking for a drink. Again, such urinary loss should not be replaced. These 16 patients literally came back from the dead.

Hypertonic sodium therapy for HN in those days was thought contraindicated but later rectified by the authorities on HN such as Professor Arieff from USA35. The anecdotal evidence of HST success in treating $\mathrm{HN}$ was reported in a letter to BMJ on an editorial on the dangers of treating $\mathrm{HN}$ at that time in 1987 as has been fully documented lately23. The effect of HST on restoring serum sodium and osmolality is reported. The effect of VO on the dilution of all serum contents, comparing symptomatic, asymptomatic and all patients is also available [21]. I think HST of $5 \% \mathrm{NaCl}$ and/ or $8.4 \% \mathrm{NaCo} 3$ works by restoring the tone of the pre-capillary sphincter and inducing diuresis. This elevates blood pressure of the arterial circulation on one hand, while restoring the capillaryISF circulation that sucks all excess fluid from the ISF space on the other. This is demonstrated so well in the physics study of the $G$ tube with its dynamic magnetic field-like fluid circulation between fluid inside $G$ tube lumen and that surrounding it in a chamber (C). I named this phenomenon the G-C circulation, akin to capillary-ISF circulation, illustrated in [?].

On final revision of this article I added more figures on the $G$ tube in circulatory system Extended data figure) for more clarity and understanding of the $G$ tube hydrodynamic. The toxic theory of glycine, sepsis with septic shock, and hemorrhage were excluded in the patho-aetiology of TURP syndrome back in 1988 in MD Thesis and in 1990 prospective study article [21] that remains as good today in 2020. The type of fluid used in resuscitation during ARDS trials is sodium-based fluid of crystalloids and colloids and/ or hydroxyethyl starch (HES) as well as blood. This is VO type 2 that also induces shock (VOS 2) causing primary ARDS and AKI36 as features of MODS. Primary VOS 2 is induced with fluids of both crystalloids and colloids such as saline, Hartmann, plasma, plasma substitutes and blood, in any combination. Primary VOS 2 has no clear serum marker like HN but hypo-albuminemia is there for VO of crystalloids but not with colloids or blood. Primary VOS 2 is much harder to detect and nearly impossible to recognize.

The hyper-volumetric state affects not only the ISF volume of subcutaneous oedema and swollen vital organs such as the heart, lung, brain and liver but also the cardiovascular system was in a state of hypervolaemia37-39. The occurrence of hypotension with 
hypervolaemia justifies calling this volumetric overload shock type 2 (VOS 2) as induced by saline-based fluids such as crystalloids and colloids. This further means that acute volume kinetics either by decrease such as severe bleeding and dehydration as well as excessive vascular volume increase by VO induces primary VOS 2 and causes primary ARDS. Surely, this VOS 2 should not be treated by further volume expansion. The endpoint of therapy here in optimizing the CVS volume in VOS 2 of ARDS in order to restore normal arterial pressure. Thus, the aim of therapy should be to lower the CVS volume down to its normal state, or even slightly below as the (-) negative sign reported by Rowan in results [7] and discussed here indicate.

For patients who are in AKI and require dialysis, the machine should be set to negative fluid balance in order to shed out the excess volume of fluids retained in the vital organs and subcutaneous ISF space. Accompanying this, the CVS volume returns to normovolaemic state. This contradicts every physicians' thought on fluid therapy in shock. Think again about it. Replacing the loss is fine to give volume that increase pressure but overdoing it may transfer hypo-volaemic shock into hyper-volaemic shock. The transition from hypo- to hyper-volaemic shock is seamless without any warning signs. The lesson was learned from the $\mathrm{G}$ tube experiment, when enclosed in a surrounding chamber (C) and connected to a circulatory model. The negative side pressure responsible for suction of fluid from $\mathrm{C}$ into the lumen of $\mathrm{G}$ creates a net negative pressure in C. Overloading the circulatory system transfers this negative $\mathrm{CP}$ into positive increasing fluid volume in $\mathrm{C}$; the equivalent of ISF oedema formation [27]. Hypotension hampers the side pressure suction force that oedema occur. To correct this, you need to raise blood pressure without worsening VO, so it is not by further volume expansion. Reduce the CVS state of hypervolaemia by diuretics that usually do not work in double or triple the normal dose, inotropes, hydrocortisone $200 \mathrm{mg}$ and the most effective as diuretic HST of $5 \% \mathrm{NaCl}$ and/or $8.4 \% \mathrm{NaCo} 3$ and the patient shall recover from early AKI and ARDS.

The centuries old debated argument on albumin versus saline, and also HES being plasma substitute, should be terminated based on the evidence from SAFE40 and FLASH41 trials that have demonstrated that neither albumen nor HES show statistically significant difference compared with saline. Again, what benefit is there to gain from such studies, if we do not implement its recommendation? The physiological study on the hind limb of sheep [27] used here as part of the evidence also demonstrated that there is no difference between albumin and saline as both induce oedema or accumulation of fluid under the cling membrane. This means that neither albumin nor HES have oncotic pressure in VIVO, which further mean that the oncotic pressure, that is one of the two force of Starling's law representing half the equation, is wrong. Thus, mathematically speaking the whole equation must also be wrong. My physics and physiological research have proved the other force of Starling's law on hydrostatic pressure is also wrong17,25,26. Thus, Starling's law is wrong on both forces. The available evidence makes the argument on albumin versus saline obsolete [35-42] and will no longer be entertained here.

What is more interesting is the result and conclusion of the study on the hind limb of sheep, representing the physiological proof reported above. It demonstrates that both albumin and saline induce oedema of the ISF space and accumulation of fluid under the cling film. This oedema occurred only when either of the two fluids is run through the vein, but not through the artery. My conclusion is that the capillaries must work as G tube not Poiseuille's tube, but it may work as Poiseuille's tube in sepsis and septic shock Volumetric overload shock type 2 (VOS2)13-16 that causes primary ARDS and/or AKI of MODS is demonstrated in this BRIDGE report. The presentation with shock occurs at the time of resuscitation of shock and trauma patients or in theatre during prolonged major surgery to anaesthetists and surgeons in the operating room and in accident and emergency department and intensive care unit (ICU) [37-39] The internists and physicians get involved about 24 hours later. So, they miss the onset of cardiopulmonary manifestations of ARDS of shock but must face the cerebral manifestations of coma, convulsion and paralysis, AKI and ARDS later, though one system of MODS may predominate- depending on the type of anaesthesia and type of fluid. The clinical picture of VOS 1 of the TURP syndrome and secondary VOS 2 causing ARDS is shown in Table 1 for comparison with that in primary VOS 2 causing ARDS.

Despite the differences between VOS 1 and VOS2 concerning fluid type and the serum marker of $\mathrm{HN}$, VO of $>7 \%$ and $>10 \%$ BW causing severe morbidity and mortality in VOS 1 is comparable with VOS2 that has VO of $>5 \mathrm{~L}(7 \%) \quad(\mathrm{P}<0.001)$ and $>10 \mathrm{~L}(15 \%)$ $(\mathrm{P}<0.001)$ as reported by Rowan7 in surviving ARDS patients with moderate to severe morbidity. Primary VOS2 does not have serum marker that is as clear as HN, but it has hypo albuminemia of saline or crystalloids of sodium-based VO. So, VO>5 L (7\%) and $>10 \mathrm{~L}(\sim 15 \%)$ are the figures that induce primary VOS 2 that cause primary ARDS morbidity. Mortality has VO of 12-14 L. This can be statistically proved on data collected immediately after resuscitation and on the day of death. Sibbald et al. [30] and Rodney and Charles [31] mentioned Starling's law in ARDS. So, it is relevant and important to discuss Starling's forces here. Other authors have expressed discontent with Starling's law criticizing it from other interesting angles such as Alphonsus and Rodseth (2014) 32 and Woodcock and Woodcock in 201228, in their review on Glycocalyx. It is also mentioned by Hahn33.34 the need for a revised paradigm to correct Starling's forces. Revised Starling equation and the glycocalyx model of trans-vascular fluid exchange: an improved paradigm for prescribing intravenous fluid therapy.

The editor of The British Journal of Anaesthesia 2012 commented on this article: "The classic Starling principle does not hold for fluid resuscitation in clinical setting." I believe this law is 
now damaged beyond repair as it is wrong on both of its forces for the reasons presented above in this BRIDGE report. I believe Starling's law is the hidden culprit that dictates the faulty rules responsible for many errors and misconceptions on fluid therapy [18] using both the conservative approach as well as liberal approach [28]. This misleads physicians into giving too much fluid [24] during the resuscitation of all types of shocks, trauma, the acutely ill patients and during prolonged major surgery. Now we have a replacement for the faulty law namely the hydrodynamic of the porous orifice (G) tube, it should be put to rest. Understanding the hydrodynamic of $\mathrm{G}$ tube provides the necessary tools that allow normalizing the circulatory hemodynamic of ARDS patient, that is the function of the pre-capillary sphincter providing the narrow orifice of $\mathrm{G}$ tube while maintaining the peripheral resistance of the arterial circulation that maintain blood pressure at a normal level. The role played by CVP in relation to the pathogenesis of VOS causing ARDS posed the question: "Does Raising the Central Venous Pressure (CVP) in Treating Shock with Fluids Induce Volumetric Overload Shocks (VOS)? This question has been positively answered with an affirmative YES [43]

Further notes on the importance of the Glycocalyx providing a smooth surface for the vascular endothelium, should the capillary be included, is demonstrated by an observation on the $G$ tube result that may apply to sepsis. Having a smooth internal surface of the $\mathrm{G}$ tube is essential for it to work properly. Any irregularities at pores of the wall disturb the negative side pressure exerted on the wall of the G tube. I think this is similar to what might happen with sepsis toxins on the endothelial wall of the capillary, plus its effect on the pre-capillary sphincter losing tone and dilating it thus transferring it from G tube to Poiseuille's tube causing arterial hypotension, i.e. hypotensive shock on one hand and inducing ISF oedema on the other. Thus, the capillary tube works as Poiseuille's tube in sepsis that induce the massive oedema of ARDS affecting vital organs and the subcutaneous tissue. Another observation on the G tube incorporated in a circulatory model is when the system is overloaded. Please see [?] Figures) for better clarity and understanding of the hydrodynamic of $\mathrm{G}$ tube.

New guidelines on fluid therapy are badly and urgently needed. Now that Professor Robert Hahn from Sweden has consented to mention his name. Robert has been a friend of mine even before our hello-goodbye meeting in 1990 when we meet at a Urological Conference in Cairo for the first and last time during the last 30 years. I have been waiting for 30 years to hear from him. Nice to hear from you again Robert who said in an email that arrived today $(17 / 02 / 2020)$ as I am putting the final touch on this BRIDGE article: "I have read your papers now. I see that you have critically read a whole lot of literature about the microcirculation and added own experiments to it, the porous orifice tube with holes that you have invented and which makes the circulation in and out of the capillary to go the opposite way. I am impressed." I reciprocate saying I am also most impressed with your research work results.

Thank you, Robert. I know of no one better qualified than Robert Hahn to head th Committee on writing new guidelines on fluid therapy. Robert Hahn was a Professor and Consultant of Anaesthesia and is now The Head of the National Fluid Academy (NFA). He is also the Editor-in-Chief of a textbook on Fluid Therapy. As mentioned above, he is the expert witness on fluid therapy. Thanks again Robert for the two pdf articles which you sent me. The authors are mentioned as they point out the importance of Glycocalyx in microcirculation and the need to revise Starling's principle. Villar et al. [44] said: "The LUNG SAFE showed a disturbingly large gap between scientific evidence and medical practice. All of these statements demand that we question the interpretations of the study's findings." One of the objectives of this BRIDGE article, as secondary endpoint, is to attempt to BRIDGE the gap between basic science and medical practice, while questioning the interpretation of studies' findings.

Just before submitting this article, I checked on how close current researchers have come to the above discussed ideas, new concepts and discoveries via PubMed $\AA$ and Google Scholar $\AA$ search engines. While I was there on $(12 / 02 / 2020)$ I searched for my reported articles such as those self-referenced here. PubMed returned 0 and Google Scholar initially returned just a few. The reason for that is Open Access journals are not listed in PubMed. We hope NBCI will soon rectify that. Later search for "Ghanem AN" on Google Scholar returned all my articles with citations. I also searched for fluid therapy and ARDS. Indeed, there is a growing body of evidence on "fluid overload" in relation to ARDS that demonstrate the importance of VO/Time concept inducing VOS causing ARDS. authors mentioned below also found a significant effect of crystalloids overload on mortality as they did the research during the first 24-48 hours from hospital admission. I have found only one study on adults' trauma patients by Jones et al. [45], and one paediatrics study by Coons et al. [46] and a remarkable review article by Schrier reported in $2010[47,48]$ that incriminate saline overload and recommend judicious use of fluid infusion during resuscitation. Schrier's review article abstract makes the finale of this BRIDGE article. In patients of these adult and paediatric trauma trials there is no sepsis involved and both were done over a period of 24 and 48 hours, respectively. Both articles detected a significant relationship of VO with morbidity and mortality of ARDS.

Jones et al. [45] reported: "Large-volume crystalloid resuscitation is associated with increased mortality and longer time ventilated. Based on this data, we recommend judicious use of crystalloids in the resuscitation of trauma patients. The conclusion by Coons et al46 was: "Early administration of high volumes of crystalloid fluid greater than $60 \mathrm{ml} / \mathrm{kg} /$ day significantly correlates with pulmonary complications, days NPO, and hospital length of stay. These results span the first $48 \mathrm{~h}$ of a patient's hospital stay and should encourage surgical care providers to exercise judicious 
use of crystalloid fluid administration in the trauma bay, ICU, and floor" Now I close this discussion with abstract of the review article by Schrier 201047. At last the BRIDGE between physics (G tube), Physiology (Hind limb of sheep), Biochemistry (Plasma electrolytes and Volume kinetics) and Medicine (VOS 1 and VOS 2 inducing the TURP syndrome and ARDS) is fully constructed and completed. "the Early Goal-Directed Therapy (EGDT) approach. These studies have demonstrated a beneficial effect on in-hospital mortality with EGDT. The Saline versus Albumin Fluid Evaluation (SAFE) randomized study in critically ill patients demonstrated no difference in survival when saline versus albumin solutions were used for resuscitation. However, a benefit of albumin has been demonstrated in a randomized study on renal function and survival in cirrhotic patients with spontaneous bacterial peritonitis. On the other hand, recent observational studies have shown a correlation between fluid overload and mortality in AKI patients whether they necessitated dialysis, or not. Moreover, the Adult Respiratory Distress Syndrome (ARDS) network performed a randomized study in critically ill patients to compare liberal versus conservative fluid administration. The liberal fluid administration group exhibited worse pulmonary function and no protection of renal function. Constancy of central venous pressure (CVP) measurements in the 12-mmHg range were observed in the liberal fluid group despite a mean increase in positive fluid balance of $7 \mathrm{~L}$, thus suggesting increased interstitial fluid accumulation leading to pulmonary congestion. The review presented here discusses these various aspects of fluid administration in critically ill patients, particularly those with AKI, and indicates the potential deleterious effects of fluid overload on lung, heart, and kidney function that could contribute to increased mortality.

\section{Conflict of Interest}

None declared by the author.

Funds received for the investigations and reporting: None declared by the author.

\section{Acknowledgements}

Many thanks go to Designer Engineer Peter Holder of Eastbourne who provided endless supply of $G$ tubes free of charge before 1985. I thank Khaled A Ghanem, MBChB for his input in editing this article, for buying me a new Laptop and Office 365. I thank Salma A Ghanem, MBChB, for help with constructing Table 2 and for payment of $\$ 200$ on my behalf to the scamsters who ripped me of it posing as conference organizers. I thank Mr Brian J Stoodley and Mr Peter Brooks, Consultant surgeons for offering me the registrar post in 1983 without attending the interview being at FRCSE examination at the time and for teaching me all the surgery I know. Many thanks go to Mr JP Ward and Mr KC Perry, Consultant Urologists, District General Hospital, Eastbourne, UK for teaching me the Urology I know, and for offering me the post of research fellow without an interview in order to do the prospective study
[19]. I also like to thank the Internet people, Google for its magic Chrome ${ }^{\circledR}$ and Google Scholar ${ }^{\circledR}$ programs, and the people behind the wonderful PubMed, NLM \& NCBI for being most helpful over the years; putting such huge amount of information at the researcher's finger's tip. I like to thank Apple ${ }^{\circledR}$ Computers for inventing the Macintosh $®$ before 1985 and Stat View $® 512+$ Statistical package; both made analyzing the data of the prospective study absolute fun 1984-1988.

\section{References}

1. Ashbaugh DG, Bigelow DB, Petty TL, Levine BE (1967) Acute respiratory distress in adults. The Lancet Saturday 12 August.

2. Montgomery AB, Stager MA, Carrico CJ, Hudson LD (1985) Causes of mortality in patients with the adult respiratory distress syndrome. Am Rev Respir Dis 132(3): 485-489.

3. Schuller D, Mitchell JP, Calandrino FS, Schuster DP (1991) Fluid balance during pulmonary edema. Is fluid gain a marker or a cause of poor outcome? Chest 100(4): 1068-1075.

4. Wioedemann HP (2006) Comparison of Two Fluid-Management Strategies in Acute Lung Injury. The National Heart, Lung, and Blood Institute Acute Respiratory Distress Syndrome (ARDS). Clinical Trials Network FACCT Trial* N Engl J Med 354: 25642575.

5. Wheeler AP, Bernard GR (2007) Acute lung injury and the acute respiratory distress syndrome: A clinical review. Lancet 369(9572): 553-1564.

6. Jacob M, Chappell D, Rehm M (2007) Clinical update: perioperative fluid management. Lancet 369(9578): 1984-1986.

7. Rowan KM, Angus DC (2017) PRISM Investigators, Early, Goal-Directed Therapy for Septic Shock - A Patient-Level Meta-Analysis. N Engl J Med 376(23): 2223-2234.

8. Huang DT, Angus DC, Amber Barnato, Scott R Gunn, John A Kellum, et al. (2013) ProCESS/ARISE/ProMISe Methodology Writing Committee, Harmonizing international trials of early goal-directed resuscitation for severe sepsis and septic shock: methodology of ProCESS, ARISE, and ProMISe. Intensive Care Med 39(10): 1760-1775.

9. Rangel-Frausto MS, Pittet D, Costigan M, Hwang T, Davis CS, et al. (1995) The natural history of the systemic inflammatory response syndrome (SIRS). A prospective study. JAMA 273(2): 117-123.

10. Angus DC, van der Poll T (2013) Severe sepsis and septic shock. N Engl J Med 369(9): 840-851.

11. Rivers E, Nguyen B, Havstad S, Julie Ressler BS, Alexandria Muzzin, et al. (2001) Early goal-directed therapy in the treatment of severe sepsis and septic shock. N Engl J Med 345(19): 1368-1377.

12. Rivers EP, Katranji M, Jaehne KA, Samantha Brown (2012) Early interventions in severe sepsis and septic shock: A review of the evidence one decade later. Minerva Anestesiol 78(6): 712-724.

13. Ghanem AN, Ghanem SA (2016) Volumetric Overload Shocks: Why Is Starling's Law for Capillary Interstitial Fluid Transfer Wrong? The Hydrodynamics of a Porous Orifice Tube as Alternative. Surgical Science $7: 245-249$.

14. Pindoria N, Ghanem SA, Ghanem KA, Ghanem AN (2017) Volumetric overload shocks in the path aetiology of the transurethral resection prostatectomy syndrome and acute dilution hyponatraemia. Integr Mol Med.

15. Ghanem Salma A, Khalid A Ghanem, Ahmed N, Ghanem (2017) Volumetric overload shocks in the patho-aetiology of the Transurethral Resection of the Prostate (TURP) Syndrome and acute dilution hyponatraemia: The clinical evidence based on prospective clinical study of 100 consecutive TURP patients. Biomed Res Clin Prac Volume 2(3): 2-7. 
16. Ghanem KA, Ghanem AN (2017) Volumetric overload shocks in the patho-aetiology of the transurethral resection prostatectomy syndrome and acute dilution hyponatraemia: The clinical evidence based on 23 case series. Basic Research Journal of Medicine and Clinical Sciences ISSN 2315-6864 6(4): pp. xx-xx.

17. Ghanem AN (2020) The Correct Replacement for the Wrong Starling's law is the Hydrodynamic of the Porous Orifice (G) Tube: The Complete Physics and physiological Evidence with Clinical Relevance and Significance. Research Article. Cardiology: Open Access Cardio Open 5(1): 1-9.

18. Ghanem AN (2018) The Adult Respiratory Distress Syndrome: Volumetric Overload Shocks in Patho-Aetiology, Correcting Errors and Misconceptions on Fluid Therapy, Vascular and Capillary Physiology. Surg. Med Open Acc J 2(2).

19. Ghanem AN (2019) Complication of Fluid Therapy Causing the Acute Respiratory Distress Syndrome: Facts and Comments. The Role of Volumetric Overload Shocks in Patho-aetiology. Archives of Urology 2(1): 21-31.

20. Essayed Yasmina Saad, Khalid A Ghanem, Salma A Ghanem, Nisha Pindoria and Ahmed N Ghanem (2019) Volumetric Overload Shocks (VOS) Resolving the Puzzle of the Transurethral Resection of the Prostate (TURP) Syndrome, Dilution Hyponatraemia (HN) and the Acute Respiratory Distress Syndrome (ARDS): The Minority Report! EC Cardiology 6(2): 109-122.

21. Ghanem AN, Ward JP (1990) Osmotic and metabolic sequelae of volumetric overload in relation to the TUR syndrome. Br J Urol 66(1): 71-78.

22. HARRISON RH, BOREN JS, ROBISON JR (1956) Dilutional hyponatraemic shock: another concept of the transurethral prostatic resection reaction. J Urol 75(1): 95-110.

23. Ghanem AN (2018) Therapy of Hyponatremia: End of Era or Minority Report? Biomed J Sci \& Tech Res 11(4)-2018. BJSTR. MS.ID.002130.

24. Ghanem AN (2020) What are Misleading Physicians into giving too much Fluid During Resuscitation of Shock and Surgery that Induces ARDS and/or AKI?" Asploro Journal of Biomedical and Clinical Case Reports 3(1): 90-98.

25. Ghanem AN (2001) Magnetic field-like fluid circulation of a porous orifice tube and its relevance to the capillary-interstitial fluid circulation: preliminary report. Med Hypotheses 56(3): 325-334.

26. Ghanem KA, Ghanem AN (2017) The proof and reasons that Starling's law for the capillary-interstitial fluid transfer is wrong, advancing the hydrodynamics of a porous orifice $(G)$ tube as the real mechanism. Blood, Heart and Circ 1(1): 17.

27. Ghanem KA, Ghanem AN (2017) The Physiological Proof that Starling's Law for the Capillary-Interstitial Fluid Transfer is wrong: Advancing the Porous Orifice (G) Tube Phenomenon as Replacement. Open Acc Res Anatomy 1(2).

28. Woodcock TE, Woodcock TM (2013) Revised Starling equation and the glycocalyx model of trans-vascular fluid exchange: An improved paradigm for prescribing intravenous fluid therapy. British Journal of Anaesthesia 108(3): 384-394.

29. Ghanem AN (2020) New Discoveries in Medicine and Physiology Originated in Urology. Surg Med Open Acc J 3(3). SMOAJ.000564.2020.

30. Sibbald WJ, Short AK, Warshawski FJ, Cunningham DG, Cheung H (1985) Thermal dye measurements of extravascular lung water in critically ill patients. Intravascular Starling forces and extravascular lung water in the adult respiratory distress syndrome. Chest 87(5): 585-592.
31. Rodney Levick, C Charles Michel (2010) Microvascular fluid exchange and the revised Starling principle. Cardiovascular Research 87: 198-210.

32. Alphonsus CS, Rodseth RN (2014) The endothelial glycocalyx: a review of the vascular barrier. Anaesthesia 69(7): 777-784.

33. Hahn RG (2017) Adverse effects of crystalloid and colloid fluids. Anaesthesiology. Intensive Ther 49(4): 303-308.

34. Hahn RG (2020) Review Article Understanding volume kinetics. Acta Anaesthesiol Scand p. 1-9.

35. Arieff AI (1986) Hyponatremia, convulsions, respiratory arrest, and permanent brain damage after elective surgery in healthy women. $\mathrm{N}$ Engl. J Med 314(24): 1529-1535.

36. Ghanem AN (2019) Preventing Renal Failure in the Critically Ill Patient: Identifying the Problems and Finding the Solutions. Ec Emergency Medicine And Critical Care 3.6.

37. Ghanem AN (2019) Fluid Resuscitation in Shock: Mini Review. Why Starling's Law Is Wrong? Surgery \& Case Studies: Open Access Journal. DOI: 10.32474/SCSOAJ.2019.02.000149.

38. Ghanem AN (2019) Shock in Polytrauma: Highlighting the Volumetric Overload Shocks and Hydrodynamic Phenomenon of the Porous Orifice (G) Tube. EC EMERGENCY MEDICINE AND CRITICAL CARE. 3(1): 29-33.

39. Ghanem AN (2020) Volumetric Overload Shocks (VOS) in Surgical Patients. Open Access J Surg 11(2): 555810.

40. SAFE Study Investigators: Simon Finfer, Rinaldo Bellomo, Suzanne McEvoy, Sing Kai Lo, SAFE Study Investigators, et al. (2006) Effect of baseline serum albumin concentration on outcome of resuscitation with albumin or saline in patients in intensive care units: analysis of data from the saline versus albumin fluid evaluation (SAFE) study. BM] 333(7577): 1044

41. Futier E, Garot M, Godet T, Matthieu Biais, Daniel Verzilli, et al. Effect of Hydroxyethyl Starch vs Saline for Volume Replacement Therapy on Death or Postoperative Complications Among High-Risk Patients Undergoing Major Abdominal Surgery: The FLASH Randomized Clinical Trial. JAMA 323(3): 225-236.

42. Ghanem AN (2019) Neither Albumen nor Capillary Pressure Work in Starling's Law, What Replacement Is There? The Hydrodynamics of The Porous Orifice Tube. Journal of Surgery Open Access: RD-SUR 10002.

43. Ghanem AN (2019) Does Raising the Central Venous Pressure (CVP) in Treating Shock with Fluids Induce Volumetric Overload Shocks (VOS)? Adv Card Res 1(5): ACR.MS.ID.000120.

44. Villar J, Schultz MJ, Kacmarek RM (2016) The LUNG SAFE: a biased presentation of the prevalence of ARDS! Crit Care 20(1): 108.

45. Jones DG, Nantais J, Rezende-Neto JB, Yazdani S, Vegas P, et al. (2018) Crystalloid resuscitation in trauma patients: deleterious effect of $5 \mathrm{~L}$ or more in the first 24h. BMC Surg 18(1): 93.

46. Coons BE, Tam S, Rubsam J, Stylianos S, Duron V (2018) High volume crystalloid resuscitation adversely affects paediatric trauma patients. J Pediatr Surg 53(11): 2202-2208.

47. Schrier RW (2010) Fluid administration in critically ill patients with acute kidney injury. Clin J Am Soc Nephrol 5(4): 733-739.

48. Guyton AC, Coleman TG (1968) Regulation of interstitial fluid volume and pressure. Annals New York Academy of Sciences 150: 537-547. 
ISSN: 2574-1241

DOI: $10.26717 /$ BJSTR.2020.29.004758

Ahmed N Ghanem. Biomed J Sci \& Tech Res

(C) (P) This work is licensed under Creative By Commons Attribution 4.0 License

Submission Link: https://biomedres.us/submit-manuscript.php

$\begin{array}{ll}\text { BIOMEDICAL } & \text { Assets of Publishing with us } \\ \text { RESEARCHES } & \text { - Global archiving of articles } \\ \text { - Immediate, unrestricted online access } \\ \text { - Rigorous Peer Review Process } \\ \text { https://biomedres.us/ }\end{array}$

